\title{
Função motora grossa de indivíduos com paralisia cerebral: implicações para a inclusão social
}

\author{
Gross motor function of cerebral palsy individuals: \\ implications for social inclusion
}

Raiane Dalmolin, ${ }^{1}$ Luciane Najar Smeha, ${ }^{1}$ Juliana Saibt Martins. ${ }^{1}$

'Centro Universitário Franciscano (UNIFRA), Santa Maria, RS, Brasil.

Recebido em: abril 2015 / Aceito em: abril 2015

jsaibt@yahoo.com.br

\section{RESUMO}

A paralisia cerebral (PC) é definida como um comprometimento motor que limita a atividade e é atribuída a distúrbios não-progressivos do encéfalo imaturo. As desordens motoras da PC são frequentemente acompanhadas por déficit cognitivo, de comunicação e de percepção sensorial, alterações comportamentais e convulsões ou a combinação desses fatores, que tornam o sujeito dependente para o auto-cuidado e mobilidade e restringem sua participação social. Objetivo: relacionar a função motora grossa de indivíduos com PC com a sua inclusão social. Método: a amostra constituiu-se por 14 pais e seus filhos com diagnóstico de PC, com idades entre um ano e 15 anos, sendo nove do sexo masculino e cinco do sexo feminino. Como instrumentos de avaliação foram utilizados um questionário semi-estruturado e a escala Gross Motor Function Classification System (GMFCS). Resultados: os resultados indicaram que a inserção de indivíduos com PC no ensino regular não dependeu de melhores níveis funcionais, também não foi encontrada diferença quanto à convivência social em lugares públicos. Considerações finais: conclui-se que o comprometimento motor, de forma isolada, não limitou a participação social dos indivíduos da amostra.

Palavras-chave: Encefalopatia; Comprometimento Motor; Participação.

\section{ABSTRACT}

Cerebral palsy $(\mathrm{CP})$ is defined as a motor impairment which limits activity, and is attributed to non-progressive disturbances during brain development. The motor disorders of CP are frequently accompanied by impaired cognition, communication, and sensory perception, behavioral abnormalities, seizure disorders, or a combination of these features, which limits the movement, making the person dependent for self-care and mobility and restricts their social participation. Objective: correlate the gross motor function of PC individuals with their social inclusion. Method: sample consisted of 14 parents and their children with CP diagnosis, aged from one to 15 years, being nine males and five females. A semi-structured questionnaire and the Gross Motor Function Classification System (GMFCS) were used. Results: results indicated that inclusion of $\mathrm{CP}$ individuals in mainstream education does not depend on better functional levels, and also no difference was found regarding social interaction in public places. Final considerations: we conclude that motor impairment alone does not limit the social participation of the individuals.

Keywords: Encephalopathy; Motor Impairment; Participation.

\section{INTRODUÇ̃̃O}

A encefalopatia crônica não evolutiva da infância ou paralisia cerebral (PC), condição sensório-motora, originalmente relatada por Little em 1862, deve-se a uma lesão cerebral de caráter não progressivo, quando este ainda é imaturo ${ }^{1,2}$, podendo ocorrer no período pré, peri ou pós-natal ${ }^{3}$. Sua classificação baseia-se em critérios como o local da lesão ${ }^{4}$, a etiologia, a distribuição topográfica e o comprometimento motor ${ }^{5}$.

A incidência da PC é estimada em dois a três casos para cada mil nascidos vivos em países desenvolvidos e, em países em desenvolvimento como o Brasil este número é superior, sendo uma das patologias mais 
prevalentes da infância ${ }^{6-9}$.

A PC é uma desordem do movimento e da postura, a qual compromete o processo de aquisição de capacidades e prejudica a execução de tarefas motoras e o desempenho do movimento ${ }^{10}$. As desordens motoras da PC são frequentemente acompanhadas por déficit cognitivo, de comunicação e de percepção sensorial, alterações comportamentais e convulsões ou a combinação desses fatores ${ }^{11}$. Assim, frequentemente os indivíduos acometidos tornam-se dependentes para o autocuidado e para a mobilidade ${ }^{12}$, o que pode limitar a sua participação social ${ }^{13}$.

O contexto social constitui outro fator que influencia diretamente no desempenho funcional dos indivíduos ${ }^{14,15}$. De fato, a funcionalidade está relacionada com a condição de saúde do indivíduo e com sua participação social, sendo influenciada pelas suas próprias características, bem como por aquelas presentes no meio ao qual ele está inserido, sendo que este pode atuar como um facilitador ou limitador da funcionalidade ${ }^{16}$. Neste sentido, a inclusão social surge com o intuito de fazer com que a sociedade reconheça a diversidade, garantindo a todos igualdade e acesso às oportunidades $^{17}$, desenvolvendo alternativas que favoreçam a participação do indivíduo no meio em que convive ${ }^{18}$. Assim, a inclusão torna-se relevante, pois a interação dos indivíduos com deficiência com o meio contribui para a construção de seus valores e organização de seu ambiente conforme suas possibilidades e as condições que o meio os proporciona ${ }^{19}$.

Nesse contexto, o objetivo desta pesquisa foi relacionar a função motora grossa de indivíduos com PC com a sua inclusão social.

\section{MATERIAL E MÉTODO}

O estudo, caracterizado como exploratório-descritivo e transversal, com abordagem quantitativa e qualitativa, foi aprovado pelo Comitê de Ética em Pesquisa com Seres Humanos do Centro Universitário Franciscano, Santa Maria - RS (CAAE 30279814.7.0000.5306).

A amostra constituiu-se por 14 pais, bem como seus filhos com diagnóstico de $\mathrm{PC}$, os quais possuíam idades entre um ano e dois meses e 15 anos, sendo nove do sexo masculino e cinco do sexo feminino. Os critérios de inclusão do estudo foram indivíduos com PC, de ambos os sexos, sem limite de idade, que realizavam tratamento fisioterapêutico no Laboratório de Ensino Prático (LEP) em Fisioterapia, assim como os seus pais. Foram excluídos do estudo os pais que não concordaram em participar da pesquisa, bem como os que estiveram impossibilitados de comparecer ao LEP, no turno da tarde. Os pais foram esclarecidos sobre os objetivos da pesquisa e solicitados a assinar o Termo de Consentimento Livre e Esclarecido.

Para avaliar o nível de participação social dos indivíduos com PC os pais foram conduzidos a uma sala reservada e de forma individual responderam a um questionário semi-estruturado com 26 perguntas, abertas e fechadas. Após, a função motora grossa dos indivíduos com PC foi avaliada através da escala Gross Motor Function Classification System (GMFCS), a qual é um instrumento que baseia-se no movimento auto-iniciado e classifica o comprometimento motor em cinco níveis diferenciados pela limitação funcional e necessidade de assistência externa. Os níveis I e II correspondem àquelas crianças com menor limitação e que conseguem deambular sem restrições; o nível III corresponde àquelas crianças que necessitam de auxílio; o nível IV/V abrange as crianças que se utilizam de tecnologia assistiva para se locomover, sendo que o nível I representa pouca ou nenhuma disfunção relativa à mobilidade e $\mathrm{V}$ totalmente dependente na mobilidade. Foi realizada a análise descritiva dos dados e estes foram apresentados em gráfico e tabela.

\section{RESULTADOS}

A tabela 1 evidencia as características demográficas e a classificação da PC em relação ao comprometimento motor da amostra. Foram avaliados 14 pais e seus 14 filhos com diagnóstico de $\mathrm{PC}$, os quais possuíam idade média de $6,7 \pm 4,8$ anos, sendo nove do sexo masculino e cinco do sexo feminino. A função motora grossa dos indivíduos foi classificada em diferentes níveis, sendo a maioria entre os níveis IV e $\mathrm{V}$ da GMFCS.

Quanto à escolaridade dos indivíduos avaliados,

Tabela 1 - Características demográficas e a classificação da PC em relação ao comprometimento motor da amostra.

\begin{tabular}{cccc}
\hline $\begin{array}{c}\text { Identificação } \\
\text { do sujeito }\end{array}$ & Sexo & Idade (anos) & Distribuição \\
topográfica
\end{tabular}


nove frequentavam a escola, seis inseridos no ensino regular. Quatro dos indivíduos avaliados já trocaram de escola pelo menos uma vez, devido a mau atendimento e /ou rejeição por parte de professores e colegas. Em relação à aceitação de seus filhos pela escola atual e pelos demais alunos, todos os entrevistados relataram ser muito boa ou ótima.

Quanto ao nível de comprometimento motor dos participantes escolares, um estava classificado no nível I da GMFCS, dois no nível III, três no nível IV e três no nível V.

Quando questionados se costumavam frequentar pracinhas, shoppings, festas com seus (a) filhos (a), oito pais responderam "frequentemente", três "algumas vezes", dois "raramente" e um "nunca". Com relação a frequentar restaurantes ou lanchonetes, sete responderam "frequentemente", três "algumas vezes", dois "raramente" e dois "nunca". Quanto à participação em eventos religiosos na comunidade quatro responderam "frequentemente", quatro "algumas vezes", dois "raramente" e um "nunca". Quando questionados se seu (a) filho (a) costuma acompanhar a família para ir ao supermercado ou para fazer algum tipo de compra, nove responderam "frequentemente", quatro "algumas vezes" e um respondeu "nunca". Costuma brincar com as outras crianças, seis "frequentemente" e um "raramente". É convidado por amigos ou colegas para participar de festas de aniversário ou outras confraternizações, seis "frequentemente", sete "algumas vezes" e um "raramente". Costuma receber em casa amigos dele da mesma faixa etária, quatro "frequentemente", três "algumas vezes", seis "raramente" e um "nunca". Costuma frequentar casa de familiares, 12 "frequentemente" e dois "algumas vezes".

Quando questionados se existiam barreiras arquitetônicas que impedem o acesso de seus filhos aos ambientes sociais, sete dos participantes responderam que não, sendo que seus filhos se classificam em diferentes níveis da GMFCS e apenas três fazem uso de cadeira de rodas. Já, para outros sete participantes, cujos filhos estão classificados nos níveis IV e V da GMFCS e cinco fazem uso de cadeira de rodas, as barreiras arquitetônicas citadas foram as pracinhas não adaptadas, falta de brinquedos especiais, ônibus sem adaptação e falta de rampas nas calçadas.

Conforme os participantes da pesquisa, os fatores que dificultam e/ou impedem a participação social de seus filhos são a pouca acessibilidade aos locais, a rejeição de pais de crianças saudáveis e a própria limitação do sujeito. Um fator que facilita é a participação frequente dos pais em ambientes sociais.

Dos indivíduos avaliados, três praticavam algum tipo de esporte, sendo que todos praticavam dança em grupo e se classificavam em diferentes níveis da GMFCS.

\section{DISCUSSÃO}

Observou-se que a maioria dos indivíduos avaliados frequentava a escola, os quais estavam inseridos principalmente no ensino regular. Neste sentido, acredita-se que a inclusão de alunos com necessidades educacionais especiais junto com outras crianças auxilie na sua socialização ${ }^{18}$, além de propiciar uma significativa melhora no desenvolvimento e na sua qualidade de vida ${ }^{20}$.

Os resultados do presente estudo mostraram que a inserção de indivíduos com PC no ensino regular não dependeu de melhores níveis funcionais, já que os indivíduos que frequentavam a escola em sua maioria estão classificados nos níveis IV e $\mathrm{V}$ da GMFCS. Em contrapartida, estudo realizado por Santos e colaboradores mostrou que a inclusão no ensino regular depende de menores níveis de comprometimento motor (níveis I e Il da GMFCS) ${ }^{20}$.

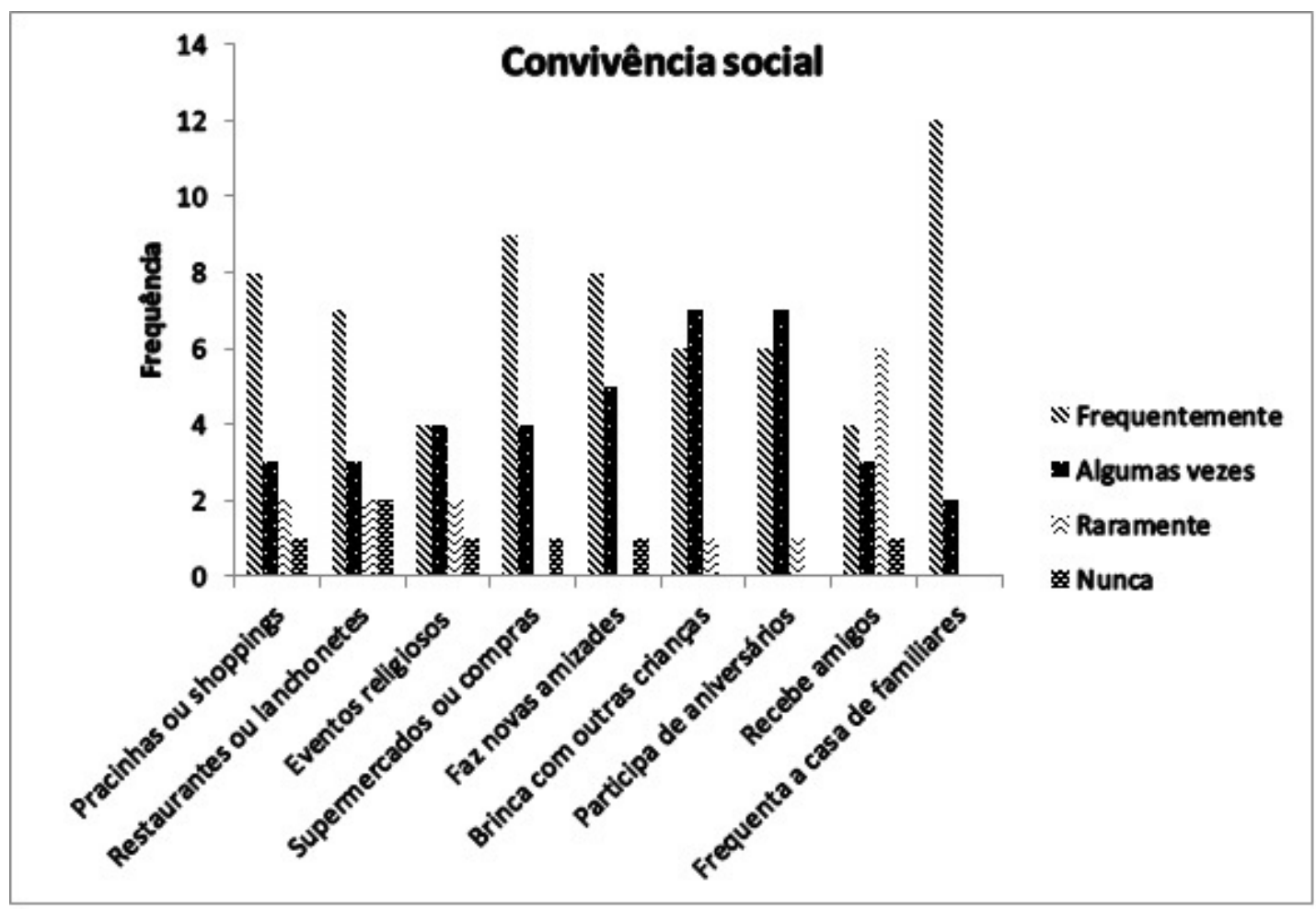

Figura 1 - Representação gráfica referente à convivência social da amostra em lugares públicos. 
Sabe-se que a inclusão escolar deve ser praticada com responsabilidade incluindo a todos, fazendo-se necessário, para isso, os recursos adequados ${ }^{21}$. No entanto, quatro dos indivíduos avaliados, os quais apresentavam comprometimento motor grave (nível $\mathrm{V}$ da GMFCS), já trocaram de escola, pelo menos uma vez, devido ao mau atendimento e/ou rejeição. Resultado semelhante foi encontrado no estudo de Souza et al, que avaliaram crianças inseridas no ensino regular e constatou que o comprometimento motor atua como um fator limitante, dificultando a abordagem da criança em sua função escolar e social ${ }^{16}$. Outro estudo que avaliou as barreiras e facilitadores do processo de inclusão em escolas de ensino regular, apontou que a falta de acessibilidade nas escolas e o despreparo dos professores constituem-se importantes barreiras no processo de insclusão ${ }^{19}$.

A escola é um ambiente fundamental para que ocorra a socialização e educação da criança durante seu desenvolvimento ${ }^{20}$. A respeito da aceitação de seus filhos pela escola atual e pelos demais alunos, todos os entrevistados relataram ser muito boa ou ótima. Neste contexto, estudo que buscou compreender a inclusão escolar de alunos com deficiência através do relato de profissionais da educação, evidenciou que alguns não encontram dificuldades em adaptar as atividades de aula para o aluno especial, já outros acham um desperdício o aluno frequentar a escola e não ser estimulado para que desenvolva suas potencialidades ${ }^{22}$.

Considerando-se o acesso dos indivíduos aos ambientes sociais como forma de inclusão destaca-se que parcela significativa dos cinemas, parques, teatros, shoppings, museus têm sido projetados ou adaptados a fim de melhorar o acesso dos indivíduos ${ }^{22}$. No entanto, ainda existem muitas barreiras arquitetônicas que impedem o acesso dos indivíduos a esses ambientes ${ }^{17}$, podendo dificultar a aquisição de suas potencialidades ${ }^{23}$.

Barreiras arquitetônicas como pracinhas não adaptadas e falta de rampas nas calçadas foram citadas pelos participantes desta pesquisa. Resultado semeIhante foi encontrado por Pereira e colaboradores, os quais mostraram que a falta de transporte urbano adaptado, difícil acesso às ruas, além de outras barreiras arquitetônicas como escassez de rampas, existência de degraus e calçadas irregulares representam dificuldades para o acesso das pessoas com deficiência ${ }^{19}$. De fato, a falta de infraestrutura adequada no meio interfere na inserção social dos indivíduos ${ }^{25}$.

Vários aspectos foram apontados pelos participantes como dificultadores da inserção social, entre eles os problemas de saúde e limitações de seus filhos. Por outro lado, a participação frequente dos pais em ambientes sociais foi apontada como um fator facilitador. Neste sentido, acredita-se que a integração das pessoas com deficiência dependa das atitudes que os membros da sociedade desempenham, podendo fazer com que elas sintam-se inseridas no meio, bem como podem tornar-se uma barreira e excluí-las do processo de integração social ${ }^{19}$.

O esporte é considerado um fator de inclusão, pois ajuda a desenvolver as potencialidades do indivíduo, de acordo com suas limitações sensoriais e físico-motoras, proporcionando melhora na qualidade de vida de seus praticantes ${ }^{26}$. No entanto a presente investigação mostrou que a minoria dos indivíduos avaliados praticava algum tipo de esporte, os quais se classificavam em diferentes níveis da GMFCS. Este resultado sugere que outros fatores, que não o comprometimento motor, possam estar envolvidos na pouca participação deles em atividades esportivas. Pesquisas recentes defendem que a participação em atividades físicas é influenciada pela idade e pela função motora grossa em adolescentes com PC, de modo que aqueles que possuem problemas motores mais graves participam menos que os demais ${ }^{27,28}$.

No que se refere à convivência social, não ficou evidenciada diferença entre os indivíduos avaliados no que se refere ao nível de comprometimento motor, visto que, os classificados em níveis IV e $\mathrm{V}$ da GMFCS costumavam frequentar pracinhas, shoppings, restaurantes, supermercados, com frequência semelhante aos classificados com menores níveis de comprometimento motor. Estudo de Vasconcelos et al encontrou que crianças com classificação motora IV e V tem maior comprometimento da função social, quando comparadas àquelas com níveis I, II e III e sugeriu que um dos fatores que pode ter contribuído para estes achados seria o fato de indivíduos classificados nos níveis IV e $\mathrm{V}$ apresentarem maiores limitações na mobilidade ${ }^{24}$. Em contrapartida, no presente estudo a maioria dos indivíduos fazia uso de cadeira de rodas e isto não os impede de frequentar lugares públicos juntamente com sua família. Neste sentido, é consenso de que o envolvimento e a disponibilidade da família é de grande importância para a inclusão social do indivíduo com PC29,30

As capacidades e dificuldades evidenciadas pela amostra deste estudo constituem-se informações importantes que deverão ser consideradas na elaboração e execução do plano de tratamento fisioterapêutico destes indivíduos.

\section{CONSIDERAÇÕES FINAIS}

Os resultados obtidos neste estudo evidenciaram que o nível de comprometimento motor parece não comprometer o processo de inclusão social, mas alguns fatores presentes no meio onde o indivíduo está inserido podem atuar como facilitadores ou dificultar sua participação social.

\section{REFERÊNCIAS}

1. Rosenbaum P, Paneth N, Leviton A, Goldstein M, Bax M. The definition and classification of cerebral palsy. Dev Med Child Neurol. 2007;49:1-44.

2. Bax $M$, Goldstein $P$, Rosenbaum $P$, Leviton $A$, Paneth $N$. Proposed definition and classification of cerebral palsy. Dev Med Child Neurol. 2005;47:571-6.

3. Krigger KW. Cerebral Palsy: An overview. Am Fam Physician. 2006;73:91-100.

4. Dzienkowski RC, Smith KK, Dillow KA, Yucha CB. Cerebral palsy: a comprehensive review. J Nurse Pratic. 1996;21:45-61.

5. Rotta NT. Paralisia cerebral, novas perspectivas terapêuticas. J Pediatric. 2002;78(supl 1):48-54.

6. BRASIL. Ministério da Saúde. Diretrizes de Atenção à Pessoa com Paralisia Cerebral. Secretaria de Atenção à 
Saúde. Departamento de Ações Programáticas Estratégicas. Brasília, 2013, 82.p.

7. Camargos ACR, Lacerda TTB, Barros TB, Silva GC, Parreiras JT, Vidal THJ. Relação entre independência funcional e qualidade de vida na paralisia cerebral. Fisioter Mov. 2012; (25):83-92

8. Pennington $L$ et al. Development of the Viking Speech Scale to classify the speech of children with cerebral palsy. Res Dev Disability. 2013;34(10):3202-10.

9. Susin FP, Bortolini V, Sukiennik R, Mancopes R, Barbosa LR. Perfil de pacientes com paralisia cerebral em uso de gastrostomia e efeito nos cuidadores. Rev CEFAC. 2012;14(5): 933-42.

10. Alcassa TC, Filipini R, Garcia JCD, Amarante ST, Adami F, Luz MCB, et al. Tetraparetic children and caregivers: featuring the profile and accessibility assistive technology. J Human Growth Dev. 2013;23(1):107-11.

11. Aisen ML, Kerkovich D, Mast J, Mulroy S, Wren TAL, Kay RM, et al. Cerebral palsy: clinical care and neurological rehabilitation. Lancet Neurol. 2011;10:844-52.

12. Mello R, Ichisato SMT, Marcon SS. Percepção da família quanto à doença e ao cuidado fisioterapêutico de pessoas com paralisia cerebral. Rev Bras Enferm. 2012;65(1):104-9.

13. Mahani MK, Karimloo M, Amisalari S. Effects of modified Adeli Suit Therapy on Improvement of Gross Motor Function in children with cerebral palsy. J Occup Ther. 2011;21:9-14.

14. Brasileiro IC, Moreira TMM, Jorge MSB, Queiroz MVO, Mont'alverne DGB. Atividades e participação de crianças com Paralisia Cerebral conforme a Classificação Internacional de Funcionalidade, Incapacidade e Saúde. Rev Bras Enferm. 2009;62(4):503-11.

15. Souza ES, Camargos ACR, Ávila $\mathrm{NCl}$, Siqueira FMS. Participação e necessidade de assistência na realização de tarefas escolares em crianças com paralisia cerebral. Fisioter Mov. 2011;24(3):409-17.

16. Pavão SL, Silva FPS, Rocha NAC. Efeito da orientação domiciliar no desempenho funcional de crianças com necessidades especiais. Motricidade. 2011;7(1):21-9.

17. Araujo JP, Schmidt A. A inclusão de pessoas com necessidades especiais no trabalho: a visão de empresas $e$ de instalações educacionais especiais na cidade de Curitiba. Rev Bras Ed Esp. 2006;12(2):241-54.

18. Almeida GC, Mancini MC, Melo APP, Drumond AF. Barreiras e facilitadores no processo de inclusão de crianças com Paralisia Cerebral em escolas de ensino regular. Cad Ter
Ocup UFSCar. 2011;19(2):203-13.

19. Pereira LMF, Caribé D, Guimarães $P$, Matsuda $D$. Acessibilidade e crianças com paralisia cerebral: a visão do cuidador primário. Fisioter Mov. 2011;24(2):299-306.

20. Santos LHC, Grissotto KP, Rodrigues DC, Bruck I. Inclusão escolar de crianças e adolescentes com paralisia cerebral: esta é uma realidade possível para todas elas em nossos dias? Rev Paul Pediatr. 2011,29(3):314-9.

21. Mazzotta MJS, D'antino MEF. Inclusão social de pessoas com deficiência e necessidades especiais: cultura, educação e lazer. Saúde Soc. 2011;20(2):377-89.

22. Gomes C, Rey FLG. Inclusão escolar: representações compartilhadas de profissionais da educação acerca da inclusão escolar. Psicol Ciênc Prof. 2007;27(3):406-17.

23. Melo FRLV, Brandão DLS, Oliveira LC, Silva LRK, Santiago LMM, Delgado RN. Estudo da acessibilidade em ambientes de lazer na cidade de Natal/RN. Licere. 2010;13(2):1-19.

24. Vasconcelos RLM, Moura TL, Campos TF, Lindquist ARR, Guerra RO. Avaliação do desempenho funcional de crianças com paralisia cerebral de acordo com níveis de comprometimento motor. Rev Bras Fisioter. 2009;13(5):390-7.

25. Milbrath VM, Soares DC, Cecagno D, Amestoy SC, Siqueira $\mathrm{HCH}$. Inserção social da criança portadora de necessidades especiais: a perspectiva materna. Cogitare Enferm. 2009;14(2):311-7.

26. Azevedo PH, Barros JF. O nível de participação do Estado na gestão do esporte brasileiro como fator de inclusão social de pessoas portadoras de deficiência. $\mathrm{R}$ Bras $\mathrm{Ci}$ e Mov. 2004;12(1):77-84.

27. Bruton LK, Barlett DJ. Description of exrcise participation of adolescents with cerebral palsy across a 4-year period. Pediatr Phys Ther. 2010;22(2):180-7.

28. Orlin MN, Palisano RJ, Charello LA, Kang LJ, Polansky M, Almasrin N, et al. Participation in home, extracurricular, and community activities among children and young people with cerebral palsy. Dev Med Child Neurol. 2010;52(2):160-6.

29. Holanda N MV, Andrade ISN. Dinâmica familiar na alimentação de crianças com paralisia cerebral. Rev Bras Pesq Saúde. 2010;23(4):374-9.

30. Brianeze ACGS, Cunha AB, Peviani SM, Miranda VCR, Tognetti VBL, Rocha NACF et al. Efeito de um programa de fisioterapia funcional em crianças com Paralisia Cerebral associado a orientações aos cuidadores: estudo preliminar. Fisioter Pesq. 2009;16(1):40-5. 